\title{
Consideraciones clínicas para fonoaudiólogos en el tratamiento de personas con COVID-19 y traqueostomía. Parte II: Mejorando la fonación para facilitar la comunicación
}

\author{
Rodrigo Tobar-Fredes a,b,*, Belén Briceño Meneses ${ }^{\mathbf{c}, \mathbf{d}}$, Macarena Venegas-Mahn e,f, Marisis Orellana Villouta ${ }^{\mathbf{e}}$, Inés \\ Fuentealba Miranda ${ }^{\mathbf{f}}$, Axel Pavez Reyes ${ }^{\text {g,h }}$, Ariela González Varas ${ }^{\mathbf{i}}$, Ying Wang ${ }^{\mathbf{j}}$, Rocío Vera-González ${ }^{\mathbf{k}, \mathbf{l}}$, Pablo Vásquez \\ Lara ${ }^{\mathbf{m}}$, Nelson Saá-Barra ", Javiera Zúñiga Reyes ñ , Gabriel Salgado Maldonado o,p, Felipe Salazar Barra q, Felipe Jiménez \\ Rojas r, Pilar Opazo-García s, Edison Gutiérrez Cifuentes t, Pamela Heusser Sagredo u \\ ${ }^{a}$ Hospital del Trabajador, Santiago, Chile \\ ${ }^{\mathbf{b}}$ Departamento de Fonoaudiologia, Universidad de Chile, Chile \\ ${ }^{\mathbf{c}}$ Hospital San Juan de Dios de Curicó, Curicó, Chile \\ d Departamento de Ciencias de la Fonoaudiologia, Universidad de Talca, Chil \\ ${ }^{\mathrm{e}}$ Hospital Clínico de la Universidad de Chile, Santiago, Chile \\ ${ }^{\mathrm{f}}$ Clinica Las Condes, Santiago, Chile \\ g Hospital de Urgencia Asistencia Pública, Santiago, Chile \\ ' Escuela de Fonoaudiologia, Universidad de Las Américas, Santiago, Chile \\ ' Hospital San José, Santiago, Chile \\ ${ }^{j}$ Hospital Hernán Henriquez Aravena, Temuco, Chile \\ ${ }^{\mathbf{k}}$ Hospital San José de Victoria, Temuco, Chile
}

\section{RESUMEN}

La enfermedad COVID-19 fue declarada pandemia por la Organización Mundial de la Salud. Su presentación más severa genera una condición que requiere tratamiento en unidades de cuidados intensivos, condición que al prolongarse en el tiempo requiere la implementación de una traqueostomía para facilitar la entrega de soporte ventilatorio invasivo. Si bien este dispositivo posee importantes ventajas que favorecen la recuperación y rehabilitación, también es cierto que genera diversas complicaciones en la comunicación de las personas, condición que se suma a los efectos propios del COVID-19 y la frecuente historia de intubación endotraqueal previa. El objetivo de este artículo es proveer orientaciones y herramientas clínicas para el tratamiento de la fonación para la comunicación en personas con traqueostomía y COVID-19. Se considera para ello las recomendaciones de la literatura existentes a la fecha, bajo un análisis pragmático y basado en nuestra experiencia de atender a más de 561 personas con esta condición. Se exponen las características de la comunicación en esta población, su tratamiento, consideraciones para el uso de técnicas específicas y orientaciones para la mejora de la calidad de vida. Siempre con un enfoque orientado al cuidado y protección de las/os usuarias/os y el equipo de salud, en particular fonoaudiólogas y fonoaudiólogos del país.

\section{Clinical considerations for speech-language pathologists in the treatment of people with COVID-19} and tracheostomy. Part II: Improving phonation to facilitate communication

\section{ABSTRACT}

The COVID-19 disease was declared a pandemic by the World Health Organization. When most severe, it generates a condition that requires treatment in intensive care units, which, when extended in time, requires implementing of a tracheostomy to facilitate invasive ventilatory support. Although ventilatory support has important advantages that favor recovery and rehabilitation, it generates various complications for patients' communication, a condition that adds to the effects of COVID-19 and the frequent history of previous endotracheal intubation. The aim of this article is to provide guidance and clinical tools for the treatment of phonation to facilitate communication in people with tracheostomy and COVID-19. For this, the recommendations of the existing available literature are considered, under a pragmatic analysis and based on our experience of treating more than 561 infected patients. The characteristics of communication in this population, its treatment, considerations for the use of specific techniques and guidelines to improve quality of life are exposed. Always with an approach oriented to the care and protection of users and the health team, in particular speech-language pathologists in the country.

\section{Palabras clave: \\ Comunicación; Voz; Traqueostomía; COVID- 19; Cuidados críticos; Fonoaudiología}

\footnotetext{
*Autor/a correspondiente: Rodrigo Tobar-Fredes

Email:ltobar@hts.cl
}

\section{Keywords: \\ Communication; Voice; Tracheostomy; COVID- 19; Critical care; Speech pathology}

Recibido: 21-09-2020

Aceptado: 11-11-2020

Publicado: 23-11-2020 
La enfermedad COVID-19 por SARS-CoV-2 fue declarada pandemia por la Organización Mundial de la Salud (OMS) el 11 de marzo de 2020. Actualmente (noviembre de 2020), el virus ha afectado mundialmente a 50.000 .000 de personas consideradas casos confirmados, incluyendo a alrededor de 1.200.000 fallecidos (Organización Mundial de la Salud [OMS], 2020). En Chile, se han registrado 518.390 casos con confirmación de laboratorio, 69.879 probables sin confirmación, y 19.382 fallecidos (Ministerio de Salud de Chile [MINSAL], 2020). Las personas con COVID-19 presentan una amplia diversidad clínica, incluso muchas son asintomáticas, transformándose en portadores y vectores de contagio (Pan et al., 2020). Otras, por contraparte, sobrellevan una presentación severa de la enfermedad, la cual genera una neumonía que deriva en un síndrome de dificultad respiratoria aguda (SDRA) (Lazzeri et al., 2020). Esta condición requiere manejo en Unidades de Cuidados Intensivos (UCI) para recibir soporte ventilatorio invasivo. Muchos de estos casos necesitan de una traqueostomía (TQT), la cual ha demostrado favorecer el destete del ventilador; mediante la disminución del trabajo respiratorio. Ello, entre otros beneficios, minimiza el riesgo de injuria glótica y facilita el despertar y la rehabilitación de la persona (Chao et al., 2020; Pierson, 2005; Shinn et al., 2019).

Sin embargo, al ser la TQT una comunicación directa con la vía aérea (VA) inferior, su manejo y manipulación contemplan un alto riesgo de contaminación para otras personas, incluidas otras también hospitalizadas y el personal de salud (Lazzeri et al., 2020). En este sentido, la contención del virus se transforma en un foco primario para los centros de salud, considerando lo altamente contagioso que es el SARS-CoV-2, el cual puede ser disgregado a través de la tos, estornudos y procedimientos generadores de aerosol (PGA) (Miles et al., 2020; Tran et al., 2012); estos últimos muy frecuentes en la atención de personas con TQT. Lo anterior implica la toma de consideraciones especiales, como son la mínima desconexión del circuito y la minimización de PGA, para evitar al máximo la propagación del virus por el flujo espiratorio de la persona (Chan et al., 2020). Dichas consideraciones van en línea con el correcto uso de los elementos de protección personal (EPP) por todo el equipo de salud (EdS) (Ferioli et al., 2020).

Las recomendaciones internacionales plantean diferentes medidas para la minimización del riesgo de aerosolización. Se incluyen el mantener el cuff insuflado y el uso de sistemas de aspiración cerrada. También se sugiere diferir los procedimientos de desinsuflado del cuff, cambio de tubo de TQT (tTQT) y decanulación, hasta que la persona sea diagnosticada como negativa para COVID-19 (Takhar et al., 2020). Sin embargo, estas recomendaciones en el contexto clínico nacional de pandemia han sido difíciles de respetar en su totalidad (Tobar-Fredes et al., 2020). Dicha situación es aún más relevante al considerar la necesidad de las personas enfermas por comunicarse con los equipos de salud y sus familias, en un contexto clínico en el cual permanecen por largos períodos de tiempo conectados a un sistema de soporte para su respiración.

Las investigaciones sobre COVID-19 aún se encuentran en desarrollo, dificultando a los clínicos el poder contar con lineamientos que permitan orientar su actuar en esta población. Como autores, respondemos a esta necesidad compartiendo la experiencia desarrollada hasta la fecha en el manejo de personas con neumonía por COVID-19 y TQT, la cual se basa en la atención de más de 561 personas afectadas en 18 centros clínicos - públicos y privados - de diferentes regiones del país. Por lo tanto, el objetivo de este artículo es proporcionar recomendaciones clínicas que permitan optimizar el tratamiento de la comunicación en esta población. Las recomendaciones que se expondrán se basan en la experiencia acumulada por los autores y la información disponible en la literatura científica a la fecha.

Para ello, se ha utilizado una metodología que es similar a la declarada en otros artículos que plantean recomendaciones para personas con COVID-19, adaptando su proceder a la urgencia con que es requerida la información (Kimura et al., 2020; Miles et al., 2020; Rovira et al., 2020). En la práctica, se ha desarrollado una revisión narrativa de la evidencia disponible para el manejo seguro y adecuado de la comunicación en personas con COVID19 y TQT. Dicha información ha sido revisada a la luz de la experiencia de los/as autores, a través de un análisis pragmático de los datos que considera las características del sistema de salud y de la pandemia en nuestro país. Así, se definen algunos lineamientos orientados a la mejora de la calidad de vida $(\mathrm{CdV})$ de las personas afectadas, mediante la mantención y/o recuperación de la comunicación; resguardando la salud de las personas hospitalizadas y del EdS.

\section{CARACTERÍSTICAS DE LA COMUNICACIÓN EN LA PERSONA CON COVID-19 Y TRAQUEOSTOMÍA}

\section{Efectos de la traqueostomía en la comunicación}

Los efectos en la comunicación de personas con COVID-19 y VA artificial son secundarios a la presencia del tubo endotraqueal y posteriormente a la implementación de una TQT (Zaga et al., 2020). Los sobrevivientes de COVID-19 que han estado intubados por períodos prolongados pueden sufrir lesiones 
cordales, desensibilización, debilidad laringo-faríngea o estenosis subglótica, así como debilidad general y des-acondicionamiento que pueden resultar en una reducción del esfuerzo o apoyo respiratorio (Castillo-Allendes et al., 2020; Zuercher et al., 2019) que se suman a afectaciones en el funcionamiento cognitivo y otros aspectos de la esfera de la salud mental (Falvey \& Ferrante, 2020). Las lesiones en cuerda vocal tras una intubación incluyen la ulceración o parálisis de estas, edema glótico o subglótico, entre otras; afectando la fonación, tanto en su intensidad como calidad vocal (Brodsky et al., 2018; Shinn et al., 2019), condición particularmente frecuente en personas que han cursado con manifestaciones severas del COVID-19 (Castillo-Allendes et al., 2020).

Cuando se instala el tubo de TQT (tTQT), se insufla el cuff para proteger las vías respiratorias y proporcionar una ventilación eficaz. Este no permite el paso de aire a través de la laringe, impidiendo la fonación. Por su parte, con el cuff desinsuflado, el propio tTQT puede obstruir notablemente la tráquea, provocando un flujo de aire deficiente, aumento de la resistencia de las vías respiratorias y un mayor trabajo respiratorio, generando una incapacidad para producir voz y por ende una adecuada comunicación verbal (Bove \& Afifi, 2017).

La limitación para comunicarse verbalmente genera un alto estrés en una persona que ya está críticamente enferma. Las personas con tTQT informan sentimientos de frustración, miedo, ansiedad e impotencia relacionados con la pérdida de la voz (Foster, 2010; Freeman, 2011). La angustia psicológica relacionada con la comunicación deficiente debido a la ventilación mecánica (VM) en la UCI está bien documentada, lo que precede a la reducida capacidad para relacionarse con los demás, aumentando el riesgo de desarrollar un trastorno de estrés postraumático tras el alta de la UCI (Pandian et al., 2020). De hecho, las personas despiertas con VM clasifican al habla como primera prioridad después de la respiración y la incomodidad física de la TQT (Morris et al., 2015).

\section{Efectos del COVID-19 en la comunicación}

Desde una perspectiva general, se ha expuesto que el uso de EPP por parte del EdS, exacerban las barreras de comunicación y sentimientos de ansiedad, pánico, miedo e impotencia (Guttormson et al., 2015). Además, en muchas personas que están en habitaciones de aislamiento por COVID-19, sin visitas y reducido contacto con el EdS; el impacto psicosocial negativo es significativo y duradero (Rosenbluth et al., 2020). Este contexto presenta una desafío único para que los/as fonoaudiólogos/as consideren estrategias y recursos de apoyo a la comunicación, incluyendo tareas de orientación y estimulación cognitiva, las cuales también contribuyen al bienestar psicológico de la persona hospitalizada y consecuentemente, disminuir el delirium (Zaga et al., 2020).

Respecto a las tareas de orientación y estimulación cognitiva, resulta muy relevante considerar que el delirium corresponde a una complicación que es común en las personas con COVID-19, siendo además marcador de un curso severo de la enfermedad, especialmente en aquellas adultas mayores y con comorbilidad neuropsiquiátrica (Kotfis et al., 2020; Ticinesi et al., 2020). El delirium, en el caso de esta población, se encuentra asociado principalmente a tres mecanismos de acción: la hipoxia derivada del SDRA, los efectos de los fármacos que permiten la sedación en personas con ventilación mecánica prolongada, y los procesos de inflamación sistémica, incluyendo estructuras cerebrales, que han sido descritos para esta enfermedad (Ramage, 2020). Este aspecto debe ser considerado dado los efectos que puede suponer la presencia de un trastorno cognitivo-comunicativo en las personas con manifestación severa de COVID-19 (Ramage, 2020), mermando aún más las capacidades comunicativas funcionales ya interferidas por los cambios fisiológicos vinculados a la presencia de VA artificial y un sistema de ventilación mecánica.

\section{INTERVENCIÓN DE LA COMUNICACIÓN EN PERSONAS CON COVID-19 Y TRAQUEOSTOMÍA}

La comunicación es fundamental para garantizar una atención segura y mejorar la CdV (Pandian et al., 2020). Para lo anterior, los/as fonoaudiólogos/as tienen un papel fundamental en la facilitación de la comunicación verbal y/o implementación no verbal a través de sistemas de comunicación alternativoaumentativa (SCAA) (Ten Hoorn et al., 2016). Considerando además el manejo del delirium como una variable clave en las fases iniciales de recuperación (Ramage, 2020; Zaga et al., 2019). En este contexto, la intervención de la comunicación en personas con VA artificial considera dos grandes aspectos:

\section{Maximizar la comunicación intrahospitalaria}

Para las personas despiertas y alertas que reciben ventilación mecánica invasiva (VMI), se recomienda incorporar SCAA para facilitar la comunicación (Zaga et al., 2019). Los/as fonoaudiólogos/as debe favorecer la educación del EdS que brinda atención a personas con COVID-19 positivas o sospechosas, respecto a los métodos de comunicación alternativoaumentativa y estrategias de apoyo comunicativas. También se 
debe considerar la provisión de recursos para personas no hispanohablantes, y personas con deficiencias cognitivas y/o comunicativas. Estos grupos tienen mayor riesgo de peores resultados de salud y disminución de la CdV (Zaga et al., 2020). Además, para aquellas personas con deficiencias cognitivocomunicativas adquiridas, como las resultantes de un delirium adquirido en UCI y posibles eventos neurológicos, requerirán la implementación de un SCAA adaptado a sus capacidades funcionales (Mao et al., 2020), así como una temprana intervención orientada a compensar aquellos aspectos cognitivocomunicativos más deficitarios, idealmente bajo un abordaje interdisciplinario (Ramage, 2020).

\section{Rehabilitación de la comunicación}

Los/as fonoaudiólogos/as realizan intervenciones que son a la vez facilitadoras y rehabilitadoras de comunicación en personas con TQT. Entre ellas se encuentran: (1) el uso de válvulas unidireccionales - también conocidas como válvulas de habla, fonación o deglución; según el objetivo para el cual es prescrita ; (2) habla ajustada al ventilador a fuga y, (3) vocalización con flujos de aire subglótico. En personas COVID-19 positivo o sospechosas, las intervenciones de comunicación deben valorarse caso a caso para evitar riesgo de exposición al SARS-CoV-2, ya que todas las técnicas mencionadas son consideradas como PGA (Zaga et al., 2020).

Paralelamente, el/la fonoaudiólogo/a debe evaluar los mecanismos implicados en la producción de la voz e iniciar técnicas que restauren el funcionamiento glótico y disminuyan la insuficiencia fonatoria; siempre maximizando las oportunidades que pueden brindar las técnicas recién mencionadas cuando se ha decidido incorporarlas en el plan de tratamiento.

En aquellos casos que se pesquisa la presencia de un trastorno cognitivo-comunicativo se ha de incorporar un plan de tratamiento enfocado en rehabilitar aquellas alteraciones cognitivas que más dificultan el funcionamiento comunicativo (Ramage, 2020), siempre rescatando las individualidades socioculturales de cada persona e idealmente bajo un modelo de intervención interdisciplinario.

Para aquellas personas que desarrollan delirium durante su estadía en UCI, la recomendación es siempre favorecer el manejo no farmacológico por sobre el farmacológico; evitando el uso de sedantes tanto como sea posible dado que pueden precipitar un empeoramiento del cuadro (Cipriani et al., 2020). Recordando además que, si bien el delirium no es inevitable, si es prevenible en aproximadamente $30-40 \%$ de los casos (Inouye et al., 2014). Las mejores prácticas de prevención incluyen, entre otras, asegurar una efectiva comunicación y orientación por parte del EdS a la persona hospitalizada, resguardar una adecuada luminosidad en la habitación, evitar la deprivación del sueño, la movilización precoz y el adecuado tratamiento el dolor (Cipriani et al., 2020).

\section{CONSIDERACIONES PARA EL USO DE TÉCNICAS ESPECÍFICAS PARA LA FACILITACIÓN DE LA COMUNICACIÓN EN PERSONAS SOSPECHOSAS O CONFIRMADAS DE COVID-19 Y TRAQUEOSTOMÍA}

\section{Inyección de flujo de aire subglótico}

En etapas temprana de la recuperación, uno de los mejores métodos para comenzar a reactivar o mantener la funcionalidad de las estructuras que permiten la emisión de la voz y el habla es la facilitación de aire hacia la VA superior, mediante la inyección de flujo de aire subglótico (Briceño et al., 2019; McGrath et al., 2019; Petosic et al., 2020). Ello debido a que, en esta etapa, las personas suelen permanecen conectadas a VMI (sin ventanas), o bien, poseen un alto riesgo de aspiración por un inadecuado manejo de sus secreciones. En este contexto, la facilitación de aire hacia la VA superior, permite la restauración de la presión positiva en la zona, siendo este un paso previo a la desinsuflación del cuff del tTQT.

La inyección de flujo de aire se realiza a través de tTQT con puerto de aspiración subglótica, dispositivos inicialmente diseñados para mejorar la higiene de las secreciones acumuladas sobre el cuff. Para facilitar la comunicación se utiliza dicho puerto para entregar flujos de aire en un rango que va desde 1 a $5 \mathrm{~L} / \mathrm{min}$, el cual ingresa para salir sobre el cuff y continuar su conducción a través de la tráquea y laringe, pasando por los pliegues vocales y saliendo por la cavidad oral, permitiendo el habla (McGrath et al., 2016; Petosic et al., 2020). Su uso también ha demostrado generar efectos positivos en la función deglutoria (McGrath et al., 2019), siendo frecuentemente la mejoría en el manejo de secreciones un objetivo previo o paralelo a la facilitación de la comunicación. Más información sobre el uso de esta técnica como una forma de rehabilitar/facilitar la deglución en personas con COVID-19 y TQT es tratada en Tobar-Fredes et al. (2020).

El procedimiento descrito, no está exento de riesgos a considerar, como la de irritación de la VA cuando se utiliza aire frío y seco, generación de enfisemas faciales o en el cuello y la hiperaducción de pliegues vocales (Calamai et al., 2018). Sin embargo, a la fecha, estos han demostrado una muy baja incidencia (Petosic et al., 2020). 
La National Tracheostomy Safety Proyect (2020) por su parte, establece directrices para fomentar la seguridad del procedimiento. Dentro de las contraindicaciones para su uso se mencionan: (1) TQT realizada hace menos de 72 horas, (2) sospecha de alteraciones estructurales en VA, (3) presencia de infección en el estoma o sangrado en VA superior, (4) mal posicionamiento de la cánula, y (5) sospecha de obstrucción de la VA superior. Como buenas practicas se declaran: (1) monitoreo profesional constante durante la inyección de flujo de aire, (2) comenzar con flujos mínimos y tiempos cortos, observando la reacción de la VA, (3) detener el procedimiento ante sospecha u observación de enfisema cutáneo, dolor o malestar en la persona, fuga de aire por el ostoma y/o presencia de signos de fatiga, cansancio motor o respiratorio.
Es frecuente que al inicio la persona tosa ante el aire inyectado, explicado por la falta de uso de las estructuras respiratorias altas, por eso es importante realizarlo con cuidado, lentamente y monitoreando la respuesta de la persona. Se recomienda que el circuito tenga conexión a un humidificador, disminuyendo los efectos del aire seco sobre tráquea y laringe. Siempre anticipando a la persona sobre el procedimiento, considerar su postura general y el posicionamiento de la cánula previo a estimular y siempre permeabilizando la VA superior antes del procedimiento (Briceño et al., 2019; McGrath et al., 2019). En personas con COVID-19 se recomienda además que el personal use todos los EPP que permita la protección ante PGA. Considerando que la sincronización del procedimiento con el uso de las EPP resulta trascendental, se esquematiza el proceder en el Diagrama 1.

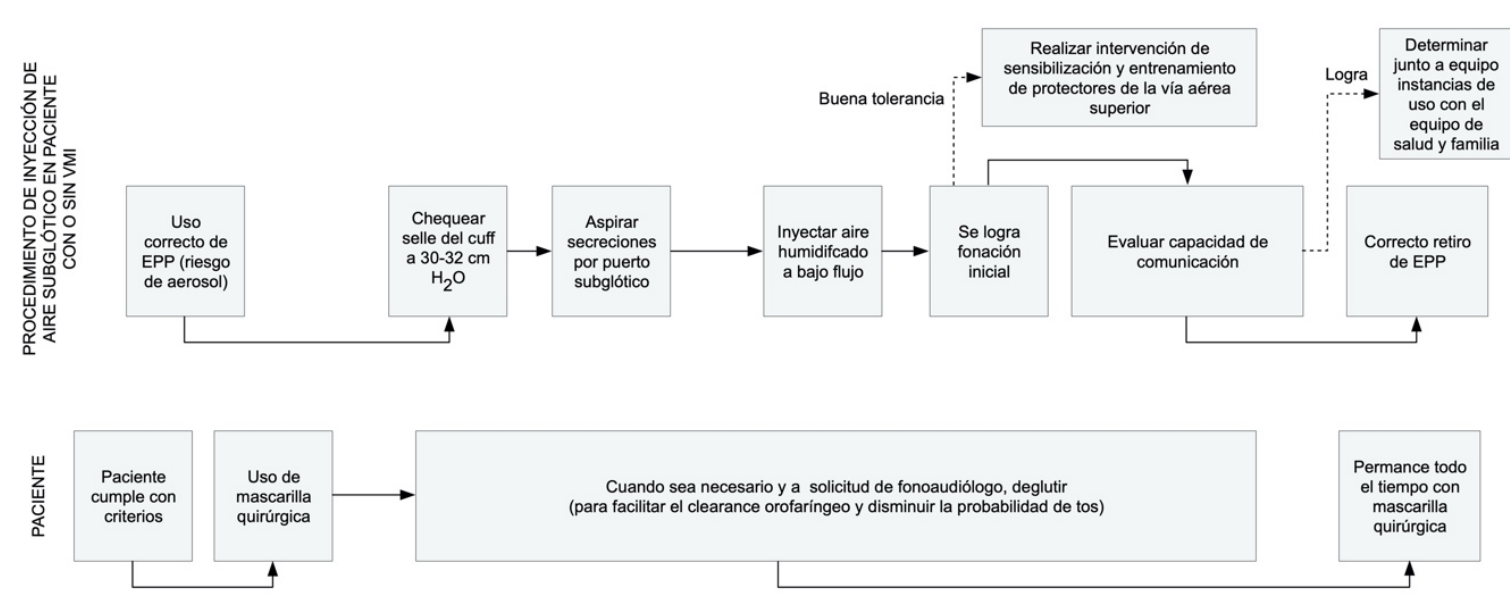

Diagrama 1. Propuesta de los autores para la sincronización entre las acciones a realizar por la persona con TQT y el procedimiento de inyección de flujo de aire subglótico, resguardando los cuidados en uso de EPP.

\section{Desinsuflado del cuff y prueba de oclusión}

El desinsuflado del cuff responde a la necesidad de evaluar la posibilidad de permitir la fuga de aire hacia VA superior para facilitar la comunicación, ecualizando las presiones que permiten una adecuada función laríngea.

En el manejo de aquellas personas con COVID-19 aún existe controversia en relación al momento en el cual es posible iniciar este procedimiento (Zaga et al., 2020). Se sugiere realizar un cuidadoso análisis de la relación riesgo-beneficio obtenido al desinsuflar el cuff, recordando que la apertura del circuito de VA artificial aumenta considerablemente el riesgo de aerosolización del virus hacia el ambiente, especialmente en aquellos sujetos que se mantienen conectados a un sistema de ventilación mecánica.

Finalmente, resulta perentorio establecer que el uso de esta técnica como una forma de facilitar la comunicación está supeditado al logro previo de una deglución funcional para el manejo de secreciones (Tobar-Fredes et al., 2020).

\section{Válvulas unidireccionales en (en persona sin ventilación mecánica invasiva)}

Las válvulas unidireccionales son ampliamente reconocidas como una herramienta útil para facilitar la comunicación en personas con TQT. Su implementación permite la recuperación fisiológica de la función laríngea, y con ello la producción de la voz. Sin 
embargo, siempre se ha de considerar que para su uso como estrategia que favorece la comunicación previamente se ha de conseguir un adecuado manejo de secreciones durante los momentos en que es implementada en el tTQT. De no ser así, y dado que requiere de la total desinsuflación del cuff, el riesgo de aspiración para la persona es alto.

En aquellas personas que es posible utilizar este tipo de facilitación de la comunicación verbal, mediante la habilitación de la voz, se ha asociado con una mejora del estado de ánimo, la perspectiva y la sensación de recuperación (Freeman-Sanderson et al., 2018). De manera particular, en el contexto de la pandemia por COVID-19, las prolongadas cuarentenas y la restricción de visitas a las personas hospitalizadas, el restablecimiento de la voz facilita su comunicación con familiares mediante sistemas como teléfono o videollamada, conectándolos con sus seres queridos, generando un incremento en el bienestar psicoemocional y favoreciendo la reducción del delirium (Cipriani et al., 2020; Rosenbluth et al., 2020; Tembo et al., 2015).

$\mathrm{Si}$ bien los beneficios comunicativos del uso de válvulas unidireccionales en personas con tTQT están ampliamente validado, se han de tomar resguardos adicionales en personas con COVID-19. Esto bajo el reconocimiento que la restauración del flujo de aire hacia la VA superior aumenta la probabilidad de que la persona tosa (Chao et al., 2009) advirtiéndose consideraciones adicionales al estándar, favoreciendo el uso seguro de estos dispositivos. Estas son aplicadas previamente durante el uso de la válvula como parte del plan de rehabilitación deglutoria (TobarFredes et al., 2020). Aquellas recomendaciones que consideramos particularmente importantes durante el uso de la válvula unidireccional con fines comunicativos son enunciadas a continuación:

- El tiempo de uso inicial de la válvula unidireccional debe ser evaluado persona a persona, considerando: tolerancia fisiológica y psicológica inicial, riesgo aspirativo, acumulación de secreciones, capacidad de automonitoreo y estabilidad respiratoria. Es recomendable que su uso inicial sea con supervisión fonoaudiológica; con un aumento progresivo y gradual de su permanencia, idealmente consensuado junto al equipo interdisciplinario.

- Si bien es cierto que la válvula unidireccional facilita la comunicación y produce beneficios fisiológicos $\mathrm{y}$ psicosociales, es necesario considerar que durante su implementación la VA queda expuesta al paso de secreciones. Por ello, el/la fonoaudiólogo/a debe evaluar este riesgo al inicio, durante y después de su uso.
- Considerando que la persona con COVID-19 se encuentra en un aislamiento riguroso y sin acompañante, es importante siempre instruir y explicar a la persona sobre el funcionamiento de válvula unidireccional, fomentando el automonitoreo y reconocimiento de signos clínicos que requieran del solicitar asistencia al EdS (voz húmeda, acumulación de secreciones, sensación de ahogo o falta de aire, cansancio, entre otras). Dejando siempre a su alcance algún sistema de llamada o timbre a ser utilizado en caso de emergencia.

- Según la posibilidad de cada centro de salud, coordinar con otros integrantes del equipo la continuidad de la supervisión e interacciones con familiares por videollamada o teléfono.

\section{CONSIDERACIONES PARA PERSONAS CON TRAQUEOSTOMÍA PRE-EXISTENTE EN CONTEXTO DE COVID-19}

La TQT siempre debe ser considerado un acceso o vía de posible contagio de COVID-19, particularmente en aquellas personas con ostoma preexistente. Recomendaciones específicas para esta población son descritas en Tobar-Fredes et al. (2020).

\section{CONSIDERACIONES GENERALES PARA LA MINIMIZACIÓN A LA EXPOSICIÓN AL VIRUS SARS- CoV-2 Y LA ATENCIÓN A PERSONAS CON COVID-19}

Dado que la totalidad de las técnicas descritas para facilitar la comunicación en personas con tTQT son consideradas como PGA, se deben considerar medidas estrictas para la prevención de la transmisión del virus SARS-CoV-2. Muchas de estas son comunes a las contempladas en el tratamiento de la deglución en personas con COVID-19 y TQT (Tobar-Fredes et al., 2020). Sobre estas se incorporan algunas adicionales que son propias de los procedimientos orientados a la comunicación:

- La intención comunicativa debe ser considerada como variable necesaria para la utilización de cualquier técnica con riesgo de aerosolización orientada hacia la facilitación de la comunicación.

- Se sugiere realizar un protocolo estandarizado de detección de impedimentos de la comunicación en todas las personas con TQT, minimizando la exposición al virus y permitiendo determinar la necesidad de una evaluación directa de la deglución, como paso previo al abordaje de la comunicación. 
- Debe existir acuerdo al interior del EdS de que la persona se encuentra en una condición clínica que va hacia la mejoría. De no ser así, se recomienda la activación de acciones de comunicación en un contexto de cuidados paliativos.

- Favorecer la comunicación de las personas en cuanto sea posible. Trabajar en coordinación con enfermería, personal auxiliar de enfermería y terapeutas ocupacionales cuando la persona tenga alguna restricción en la movilidad de extremidades superiores, para implementar un SCAA o cuando requieran algún grado de asistencia para su uso. Para mayores especificaciones y orientaciones al EdS respecto al uso de los SCAA, se recomienda revisar Vera-González et al. (2020).

- En todas las personas hospitalizadas, incluso aquellas que requieren de cuidados paliativos, siempre considerar estrategias que favorezcan el bienestar. Se debe privilegiar el confort de la persona y las necesidades específicas de cada individuo, considerando: (1) facilitar la comunicación mediante válvulas unidireccionales o flujo de aire subglótico al menos por algunos breves períodos, equilibrando la seguridad de la intervención con los beneficios psicoemocionales para la persona, (2) implementar SCAA centrado en las necesidades específicas del individuo, y (3) capacitar al EdS, cuidador(a) y familia en la técnica que se ha implementado como facilitador de la comunicación.

\section{COMENTARIOS DE LOS AUTORES}

La perdida o alteración en la comunicación de los usuarios con TQT en contexto de la pandemia COVID-19 aumenta el mal pronóstico y entorpece la rehabilitación de otras especialidades. Por tanto, un trabajo temprano y coordinado favorece y facilita una recuperación funcional más rápida.

Un consenso sobre el manejo de eyección de presiones de aire por vía subglotica y uso de válvula unidireccional es fundamental para beneficiar de forma segura la función comunicativa del usuario y otras competencias asociadas. En este documento se plantean aquellas que han sido consensuadas por los autores, sin embargo, es necesario proseguir con este trabajo permitiendo la generación de guías nacionales.

Es importante recalcar que las técnicas, sus procedimientos y los criterios de su uso no solo deben se concordantes con el análisis costo-beneficio previamente discutido con el equipo de rehabilitación, sino además bien ejecutadas para así evitar riesgos o lesiones en el usuario. Para ello, las recomendaciones descritas en este artículo entregan orientaciones en esa línea, pero que siempre deben ser adecuadas y flexibilizadas a las características de cada persona y centro de salud.

Finalmente, invitamos a compartir cada una de las experiencias que se han generado a propósito de esta pandemia, favoreciendo el desarrollo del conocimiento y permitiendo mejorar continuamente la práctica clínica fonoaudiológica. Así mismo, se recomienda la recolección de la información disponible en cada centro, con el fin de poder precisar a futuro aún más las orientaciones entregadas en este documento. Recordando que este es una primera aproximación basada en la evidencia disponible a la fecha y experiencias internacionales; todas analizada a la luz de la experiencia desarrollada por los autores en la atención de personas con COVID-19 y TQT en nuestro país.

\section{REFERENCIAS}

Bove, M. J., \& Afifi, M. S. (2017). Tracheotomy procedure. En L. L. Morris \& M. S. Afifi (Eds.), Tracheostomies: The Complete Guide. Springer Publishing.

Briceño, B., Márques, S., Pinto, P., \& Bravo, C. (2019). Protocolo de intervención Interdisciplinaria para la decanulación de personas con traqueostomia desconectadas de ventilación mecánica. En M. P. Moya, F. Susanibar, \& C. Valdés (Eds.), Evaluación e Intervención Logopédica en Motricidad Orofacial y áreas afines. Giunti EOS.

Brodsky, M. B., Levy, M. J., Jedlanek, E., Pandian, V., Blackford, B., Price, C., Cole, G., Hillel, A. T., Best, S. R., \& Akst, L. M. (2018). Laryngeal Injury and Upper Airway Symptoms After Oral Endotracheal Intubation With Mechanical Ventilation During Critical Care: A Systematic Review. Critical Care Medicine, 46(12), 2010-2017. https://doi.org/10.1097/CCM.0000000000003368

Calamai, I., Giuntini, R., Tomeo, F., \& Spina, R. (2018). Sudden appearance of neck and face emphysema during above cuff vocalisation. Intensive Care Medicine, 44(11), 1951-1952. https://doi.org/10.1007/s00134-018-5233-6

Castillo-Allendes, A., Contreras-Ruston, F., Cantor-Cutiva, L. C., Codino, J., Guzman, M., Malebran, C., Manzano, C., Pavez, A., Vaiano, T., Wilder, F., \& Behlau, M. (2020). Voice Therapy in the Context of the COVID-19 Pandemic: Guidelines for Clinical Practice. Journal of Voice. https://doi.org/10.1016/j.jvoice.2020.08.001

Chan, J. Y. K., Wong, E. W. Y., \& Lam, W. (2020). Practical Aspects of Otolaryngologic Clinical Services During the 2019 Novel Coronavirus Epidemic: An Experience in Hong Kong. JAMA Otolaryngology-- Head \& Neck Surgery, 146(6), 519-520. https://doi.org/10.1001/jamaoto.2020.0488

Chao, C. Y. H., Wan, M. P., Morawska, L., Johnson, G. R., Ristovski, Z. D., Hargreaves, M., Mengersen, K., Corbett, S., Li, Y., Xie, X., \& Katoshevski, D. (2009). Characterization of expiration air jets and droplet size distributions immediately at the mouth opening. Journal of Aerosol Science, 40(2), 122-133. https://doi.org/10.1016/j.jaerosci.2008.10.003

Chao, T. N., Harbison, S. P., Braslow, B. M., Hutchinson, C. T., Rajasekaran, K., Go, B. C., Paul, E. A., Lambe, L. D., Kearney, J. J., Chalian, A. A., Cereda, M. F., Martin, N. D., Haas, A. R., Atkins, J. H., \& Rassekh, C. H. (2020). Outcomes After Tracheostomy in COVID-19 Patients. Annals of Surgery, 272(3), e181-e186. https://doi.org/10.1097/SLA.0000000000004166 
Cipriani, G., Danti, S., Nuti, A., Carlesi, C., Lucetti, C., \& Di Fiorino, M. (2020). A complication of coronavirus disease 2019: Delirium. Acta Neurologica Belgica, 120(4), 927-932. https://doi.org/10.1007/s13760-020-01401-7

Falvey, J. R., \& Ferrante, L. E. (2020). Flattening the disability curve: Rehabilitation and recovery after COVID-19 infection. Heart \& Lung, 49(5), 440441. https://doi.org/10.1016/j.hrtlng.2020.05.001

Ferioli, M., Cisternino, C., Leo, V., Pisani, L., Palange, P., \& Nava, S. (2020). Protecting healthcare workers from SARS-CoV-2 infection: Practical indications. European Respiratory Review, 29(155). https://doi.org/10.1183/16000617.00682020

Foster, A. (2010). More than nothing: The lived experience of tracheostomy while acutely ill. Intensive \& Critical Care Nursing, 26(1), 33-43. https://doi.org/10.1016/j.icen.2009.09.004

Freeman, S. (2011). Care of adult patients with a temporary tracheostomy. Nursing Standard, 26(2), 49-56. https://doi.org/10.7748/ns2011.09.26.2.49.c8706

Freeman-Sanderson, A. L., Togher, L., Elkins, M., \& Kenny, B. (2018). Quality of life improves for tracheostomy patients with return of voice: A mixed methods evaluation of the patient experience across the care continuum. Intensive \& Critical Care Nursing, 46, 10-16. https://doi.org/10.1016/j.iccn.2018.02.004

Guttormson, J. L., Bremer, K. L., \& Jones, R. M. (2015). "Not being able to talk was horrid": A descriptive, correlational study of communication during mechanical ventilation. Intensive \& Critical Care Nursing, 31(3), 179-186. https://doi.org/10.1016/j.iccn.2014.10.007

Inouye, S. K., Westendorp, R. G. J., \& Saczynski, J. S. (2014). Delirium in elderly people. Lancet, 383(9920), 911-922. https://doi.org/10.1016/S01406736(13)60688-1

Kimura, Y., Ueha, R., Furukawa, T., Oshima, F., Fujitani, J., Nakajima, J., Kaneoka, A., Aoyama, H., Fujimoto, Y., \& Umezaki, T. (2020). Society of swallowing and dysphagia of Japan: Position statement on dysphagia management during the COVID-19 outbreak. Auris Nasus Larynx, 47(5), 715-726. https://doi.org/10.1016/j.anl.2020.07.009

Kotfis, K., Williams Roberson, S., Wilson, J. E., Dabrowski, W., Pun, B. T., \& Ely, E. W. (2020). COVID-19: ICU delirium management during SARS-CoV-2 pandemic. Critical Care (London, England), 24(1), 176. https://doi.org/10.1186/s13054-020-02882-x

Lazzeri, M., Lanza, A., Bellini, R., Bellofiore, A., Cecchetto, S., Colombo, A., D’Abrosca, F., Del Monaco, C., Gaudiello, G., Paneroni, M., Privitera, E., Retucci, M., Rossi, V., Santambrogio, M., Sommariva, M., \& Frigerio, P. (2020). Respiratory physiotherapy in patients with COVID-19 infection in acute setting: A Position Paper of the Italian Association of Respiratory Physiotherapists (ARIR). Monaldi Archives for Chest Disease $=$ Archivio Monaldi Per Le Malattie Del Torace, 90(1). https://doi.org/10.4081/monaldi.2020.1285

Mao, L., Jin, H., Wang, M., Hu, Y., Chen, S., He, Q., Chang, J., Hong, C., Zhou, Y., Wang, D., Miao, X., Li, Y., \& Hu, B. (2020). Neurologic Manifestations of Hospitalized Patients With Coronavirus Disease 2019 in Wuhan, China. JAMA Neurology, 77(6), 683-690. https://doi.org/10.1001/jamaneurol.2020.1127

McGrath, B. A., Wallace, S., Wilson, M., Nicholson, L., Felton, T., Bowyer, C., $\&$ Bentley, A. M. (2019). Safety and feasibility of above cuff vocalisation for ventilator-dependant patients with tracheostomies. Journal of the Intensive Care Society, 20(1), 59-65. https://doi.org/10.1177/1751143718767055
McGrath, B., Lynch, J., Wilson, M., Nicholson, L., \& Wallace, S. (2016). Above cuff vocalisation: A novel technique for communication in the ventilatordependent tracheostomy patient. Journal of the Intensive Care Society, 17(1), 1926. https://doi.org/10.1177/1751143715607549

Miles, A., Connor, N. P., Desai, R. V., Jadcherla, S., Allen, J., Brodsky, M., Garand, K. L., Malandraki, G. A., McCulloch, T. M., Moss, M., Murray, J., Pulia, M., Riquelme, L. F., \& Langmore, S. E. (2020). Dysphagia Care Across the Continuum: A Multidisciplinary Dysphagia Research Society Taskforce Report of Service-Delivery During the COVID-19 Global Pandemic. Dysphagia. https://doi.org/10.1007/s00455-020-10153-8

Ministerio de Salud de Chile [MINSAL]. (2020). Informe epidemiológico $N^{0} 66$ Enfermedad por SARS-CoV-2 (COVID-19). MINSAL. https://www.minsal.cl/wpcontent/uploads/2020/11/Informe-Epidemiologia-66.pdf

Morris, Linda L., Bedon, A. M., McIntosh, E., \& Whitmer, A. (2015). Restoring Speech to Tracheostomy Patients. Critical Care Nurse, 35(6), 13-28. https://doi.org/10.4037/ccn2015401

National Tracheostomy Safety Proyect. (2020). ACV - Above cuff vocalization. Swallowing \& communication (adults) http://www.tracheostomy.org.uk/healthcare-staff/vocalisation/acv-above-cuffvocalisation.

Organización Mundial de la Salud [OMS]. (2020). Coronavirus disease (COVID19) outbreak situation. https://www.who.int/emergencies/diseases/novelcoronavirus-2019

Pan, X., Chen, D., Xia, Y., Wu, X., Li, T., Ou, X., Zhou, L., \& Liu, J. (2020). Asymptomatic cases in a family cluster with SARS-CoV-2 infection. The Lancet. Infectious Diseases, 20(4), 410-411. https://doi.org/10.1016/S14733099(20)30114-6

Pandian, V., Cole, T., Kilonsky, D., Holden, K., Feller-Kopman, D. J., Brower, R., \& Mirski, M. (2020). Voice-Related Quality of Life Increases With a Talking Tracheostomy Tube: A Randomized Controlled Trial. The Laryngoscope, 130(5), 1249-1255. https://doi.org/10.1002/lary.28211

Petosic, A., Viravong, M. F., Martin, A. M., Nilsen, C. B., Olafsen, K., \& Berntzen, H. (2020). Above cuff vocalisation (ACV): A scoping review. Acta Anaesthesiologica Scandinavica. https://doi.org/10.1111/aas.13706

Pierson, D. J. (2005). Tracheostomy and weaning. Respiratory Care, 50(4), 526533.

Ramage, A. (2020). Potential for Cognitive Communication Impairment in COVID-19 Survivors: A Call to Action for Speech-Language Pathologists. American Journal of Speech-Language Pathology, 29(4), 1821-1832. https://doi.org/10.1044/2020_AJSLP-20-00147

Rosenbluth, G., Good, B. P., Litterer, K. P., Markle, P., Baird, J. D., Khan, A., Landrigan, C. P., Spector, N. D., Patel, S. J., \& SHM I-PASS SCORE Study Group. (2020). Communicating Effectively With Hospitalized Patients and Families During the COVID-19 Pandemic. Journal of Hospital Medicine, 15(7), 440-442. https://doi.org/10.12788/jhm.3466

Rovira, A., Dawson, D., Walker, A., Tornari, C., Dinham, A., Foden, N., Surda, P., Archer, S., Lonsdale, D., Ball, J., Ofo, E., Karagama, Y., Odutoye, T., Little, S., Simo, R., \& Arora, A. (2020). Tracheostomy care and decannulation during the COVID-19 pandemic. A multidisciplinary clinical practice guideline. European Archives of Oto-Rhino-Laryngology, 1-9. https://doi.org/10.1007/s00405-02006126-0 
Shinn, J. R., Kimura, K. S., Campbell, B. R., Sun Lowery, A., Wootten, C. T., Garrett, C. G., Francis, D. O., Hillel, A. T., Du, L., Casey, J. D., Ely, E. W., \& Gelbard, A. (2019). Incidence and Outcomes of Acute Laryngeal Injury After Prolonged Mechanical Ventilation. Critical Care Medicine, 47(12), 1699-1706. https://doi.org/10.1097/CCM.0000000000004015

Takhar, A., Walker, A., Tricklebank, S., Wyncoll, D., Hart, N., Jacob, T., Arora, A., Skilbeck, C., Simo, R., \& Surda, P. (2020). Recommendation of a practical guideline for safe tracheostomy during the COVID-19 pandemic. European Archives of Oto-Rhino-Laryngology: Official Journal of the European Federation of Oto-Rhino-Laryngological Societies (EUFOS): Affiliated with the German Society for Oto-Rhino-Laryngology - Head and Neck Surgery, 277(8), 2173-2184. https://doi.org/10.1007/s00405-020-05993-x

Tembo, A. C., Higgins, I., \& Parker, V. (2015). The experience of communication difficulties in critically ill patients in and beyond intensive care: Findings from a larger phenomenological study. Intensive \& Critical Care Nursing, 31(3), 171178. https://doi.org/10.1016/j.iccn.2014.10.004

Ten Hoorn, S., Elbers, P. W., Girbes, A. R., \& Tuinman, P. R. (2016). Communicating with conscious and mechanically ventilated critically ill patients: A systematic review. Critical Care (London, England), 20(1), 333. https://doi.org/10.1186/s13054-016-1483-2

Ticinesi, A., Cerundolo, N., Parise, A., Nouvenne, A., Prati, B., Guerra, A., Lauretani, F., Maggio, M., \& Meschi, T. (2020). Delirium in COVID-19: Epidemiology and clinical correlations in a large group of patients admitted to an academic hospital. Aging Clinical and Experimental Research, 1-8. https://doi.org/10.1007/s40520-020-01699-6

Tobar-Fredes, R., Briceño, B., Fuentealba, I., Orellana, M., Venegas-Mahn, M., Pavez, A., Vásquez, P., Wang, Y., González, A., Salgado, G., Saá-Barra, N., Zúñiga, J., Gutiérrez, E., Vera-González, R., Salazar, F., Jiménez, F., OpazoGarcía, P., \& Heusser, P. (2020). Consideraciones clínicas para fonoaudiólogos en el tratamiento de personas con COVID-19 y traqueostomía. Parte I: Deglución. Revista Chilena de Fonoaudiología, 19, 1-12. https://doi.org/10.5354/07194692.2020 .60185

Tran, K., Cimon, K., Severn, M., Pessoa-Silva, C. L., \& Conly, J. (2012). Aerosol generating procedures and risk of transmission of acute respiratory infections to healthcare workers: A systematic review. PloS One, 7(4), e35797. https://doi.org/10.1371/journal.pone.0035797

Vera-González, R., Márquez-Ramírez, O., \& Tobar-Fredes, R. (2020). Recomendaciones para facilitar la comunicación en personas con vía aérea artificial y COVID-19. Sociedad Chilena de Medicina Intensiva. https://www.medicina-

intensiva.cl/site/covid/guias/Recomendaciones_Facilitacion Comunicacion.pdf

Zaga, C., Berney, S., \& Vogel, A. P. (2019). The Feasibility, Utility, and Safety of Communication Interventions With Mechanically Ventilated Intensive Care Unit Patients: A Systematic Review. American Journal of Speech-Language Pathology, 28(3), 1335-1355. https://doi.org/10.1044/2019_AJSLP-19-0001

Zaga, J., Pandian, V., Brodsky, M., Wallace, S., Cameron, T., Chao, C., Orloff, L. A., Atkins, N., McGrath, B., Lazarus, C., Vogel, A., \& Brenner, M. (2020). Speech-Language Pathology Guidance for Tracheostomy During the COVID-19 Pandemic: An International Multidisciplinary Perspective. American Journal of $\begin{array}{lll}\text { Speech-Language } \quad \text { Pathology, } & \text { 29(3), }\end{array}$ https://doi.org/10.1044/2020_AJSLP-20-00089

Zuercher, P., Moret, C. S., Dziewas, R., \& Schefold, J. C. (2019). Dysphagia in the intensive care unit: Epidemiology, mechanisms, and clinical management. Critical Care (London, England), 23(1), 103. https://doi.org/10.1186/s13054-019$2400-2$ 